\title{
Carbon nanotube based sensors and fluctuation enhanced sensing
}

\author{
Ákos Kukovecz,, , Dániel Molnár², Krisztián Kordás ${ }^{3}$, Zoltán Gingl ${ }^{4}$, Hannu Moilanen ${ }^{5}$, Róbert Mingesz ${ }^{4}$, \\ Zoltán Kónya $^{1}$, Jani Mäklin ${ }^{3}$, Niina Halonen ${ }^{3}$, Géza Tóth $^{3}$, Henrik Haspel ${ }^{1}$, Péter Heszler ${ }^{2}$, Melinda Mohl $^{1}$, \\ András Sápi ${ }^{1}$, Siegmar Roth ${ }^{6}$, Robert Vajtai ${ }^{7}$, Pulickel M. Ajayan ${ }^{7}$, Yann Pouillon $^{8}$, Angel Rubio $^{8}$ and \\ Imre Kiricsi ${ }^{1}$ \\ ${ }^{1}$ Department of Applied and Environmental Chemistry, University of Szeged, Rerrich B. tér 1, H-6720 Szeged, Hungary \\ ${ }^{2}$ Department of Optics and Quantum Electronics, University of Szeged, Dóm tér 9, H-6720 Szeged, Hungary \\ ${ }^{3}$ Microelectronics and Materials Physics Laboratories, and EMPART Research Group of Infotech Oulu, University of Oulu, P.O. Box \\ 8500, FIN-90014 University of Oulu, Finland \\ ${ }^{4}$ Department of Experimental Physics, University of Szeged, Dóm tér 9, H-6720, Szeged, Hungary \\ ${ }^{5}$ Laserprobe LP Ltd. Jussintie 6, FIN-90800 Oulu, Finland \\ ${ }^{6}$ Max Planck Institute for Solid State Research, Heisenbergstr. 1, D-70569 Stuttgart Germany \\ ${ }^{7}$ Department of Mechanical Engineering and Materials Science, Rice University, Houston, Texas 77251-1892, USA \\ ${ }^{8}$ Department of Material Physics, University of Pais Vasco, Avenida de Tolosa, 72, 20018 Donostia-San Sebastian, Spain
}

Received ZZZ, revised ZZZ, accepted ZZZ

Published online ZZZ (Dates will be provided by the publisher.)

PACS 05.40.-a, 07.07.Df, 61.48.De, 73.61.Wp, 73.63.Rt

*Corresponding author: e-mail kakos@chem.u-szeged.hu, Phone: +36 62544 620, Fax: +36 62544619

\begin{abstract}
An inkjet-printed thin film of multi-wall carbon nanotubes is used as a gas sensor in a four-probe measurement setup. The novelty of the approach is that the sensor information is extracted from the noise of the dc resistance data using Fluctuation Enhanced Sensing (FES). We investigate the effects of measurement duration, gate voltage and frequency window on the chemical selectivity of the MWCNT-FES sensor. The selectivity of the device is superior to those of conventional gas sensors, and preliminary experiments indicate that it may also be possible to extract quantitative information from the noise.
\end{abstract}

Introduction Gas sensing is a heavily competitive field where real-life applications of nanoscale materials are already commercialized today [1-3]. Developments in gas sensing need to offer radical performance increases over the existing solutions in order to attract the attention of potential manufacturers and customers. The performance of any sensor is determined primarily by its sensitivity and selectivity $[4,5]$. Nowadays, sensors measuring in the subppm concentration range are fairly common [6]. On the other hand, improving the selectivity of a sensor can be

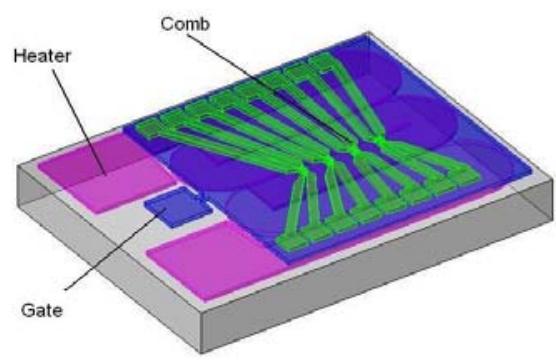

Structure of the sensor chip used in this work. The device can carry out four parallel four-probe measurements with a controlled gate voltage and sensor film temperature.

Copyright line will be provided by the publisher

very challenging. The traditional approach is to functionalize the sensing layer in a way that promotes the selective adsorption of a target molecule, and combine several differently functionalized sensors into an array to achieve selectivity at the device level [7].

Carbon nanotubes (CNTs) have been extensively researched as gas sensor materials in conventional resistive [8], transistor gain measurement [9] and even in field emission current measurement [10] configurations. Until now, even CNTs were unable to offer notable selectivity 
improvements because CNT sensorics research was focused on conventional measurement techniques.

We have reported recently that by analysing the noise of a sensor device instead of its primary output signal (e.g. dc resistance) it is possible to differentiate between four different analyte molecules at the single sensor level [11]. This phenomenon is known as fluctuation enhanced sensing (FES) [12]. The physics behind FES is that the molecular level contacts between the individual building blocks in any sensor layer (the carbon nanotubes in our case) are slightly different from one another and consequently, they offer "specific" adsorption geometries for all sorts of analytes even without functionalization. However, since it is impossible to read out individual CNT-CNT contacts in a nanotube film, the analyte-specific information is usually lost for the observer as it manifests only in the noise of the sensor signal. FES is the method of extracting information from the sensor noise.

In our earlier reports we have demonstrated the feasibility of the CNT FES concept [13]. In the present report we discuss the possibilities of improving FES selectivity by manipulating the operational variables of a working FES sensor device.
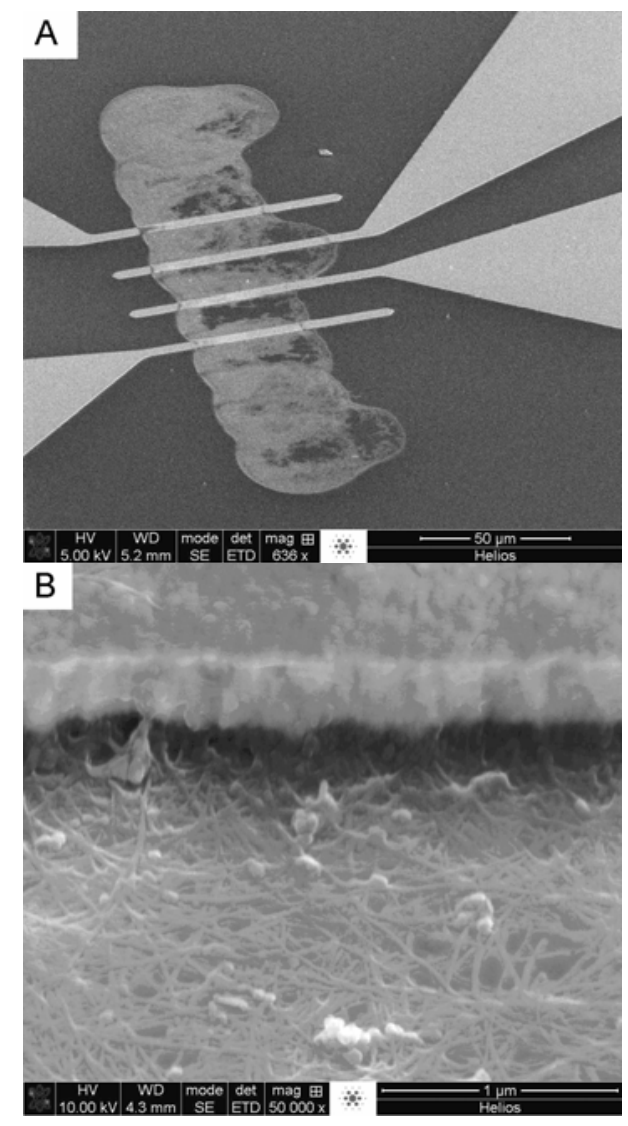

Figure 1 SEM images of the printed MWCNT sensor film (A) and a close-up of the MWCNT-electrode interface region $(\mathrm{B})$
2. Experimental Carbon nanotube ink was prepared by sonicating $2.0 \mathrm{mg}$ unfunctionalized multi-wall carbon nanotube material in $15 \mathrm{ml}$ of water for $1 \mathrm{~h}$. The ink was centrifuged at $3500 \mathrm{rpm}$ for $30 \mathrm{~min}$. Then the supernatant solution was collected and centrifuged again for $15 \mathrm{~min}$. The concentration of nanotubes in the last supernatant solution was $\approx 0,06 \mathrm{mg} \cdot \mathrm{mL}^{-1}$. The ink was loaded into a new printer cartridge and deposition was carried out using a DIMATIX Fujifilm printer [14]. The sensor film was printed over the four electrode fingers in a $200 \mu \mathrm{m}$ long line pattern using 9 drops. As illustrated in Figure 1. the diameter of the drops was approximately $35 \mu \mathrm{m}$ while the distance between the drops was $25 \mu \mathrm{m}$. The target dc resistance of the dried sensor film was $100 \mathrm{k} \Omega$.

Gas sensing measurements were performed in artificial air atmosphere at $25{ }^{\circ} \mathrm{C}$ using a custom sensor test equipped with special, low noise electronics. Details of the measurement procedure were reported previously [8]. It is enough to mention here that the primary output of the sensor device is the dc resistance of the carbon nanotube film as measured in a four-point measurement setup. In the FES data analysis process the noise of the signal is extracted, amplified, and then the power spectral density (PSD) of the noise is calculated. Finally, the PSD is subjected to Principal Component Analysis (PCA) and the results are displayed in a PCA map which is easily understandable by humans: clustering of independent measurements performed on the same analyte indicate high selectivity, while a random distribution of observations is the sign of poor sensor selectivity.

\section{Results and discussion}

3.1 Effects of PSD averaging The power spectral density (PSD) that characterizes the stationary fluctuations of the sensor device - itself is "noisy" i.e., has a signal to noise ratio (SNR). The PSD also has a spectral window, its upper limit is half of the sampling frequency $\left(f_{s}\right)$ and its lower limit is the reciprocal value of the data acquisition time $\left(t_{a}\right)$. Then a PSD function can be derived from the $t_{a} f_{s}$ number of acquired data points by fast Fourier transform, see Figure $2 \mathrm{a}$. It can be seen that the spectra are very noisy. To decrease the noise (increasing SNR), one can repeat the measurement by $N_{a}$ times (noting as number of averaging), i.e. the total measurement time $\left(t_{m}\right)$ and total number of acquired data points $\left(n_{d}\right)$ for the data acquisition will be $t_{m}=$ $N_{a} t_{a}$ and $n_{d}=N_{a} t_{a} f_{s}$, respectively. PSD spectra with $N_{a}=$ 10 and 100 are depicted in Figure 2b,c showing significantly increased SNR. However, increasing $N_{a}$ results in an increased $t_{m}$, therefore it is important to determine the $N_{a}$ values to differentiate the analytes.

The SNR increases as $N_{a}$ is increased (see Figure 2) and this makes possible to differentiate between the applied test gases, see Figure 2, bottom row. When $N_{a}=1$, no discrimination was possible using principal component analysis (PCA) as a pattern recognition method taking the 

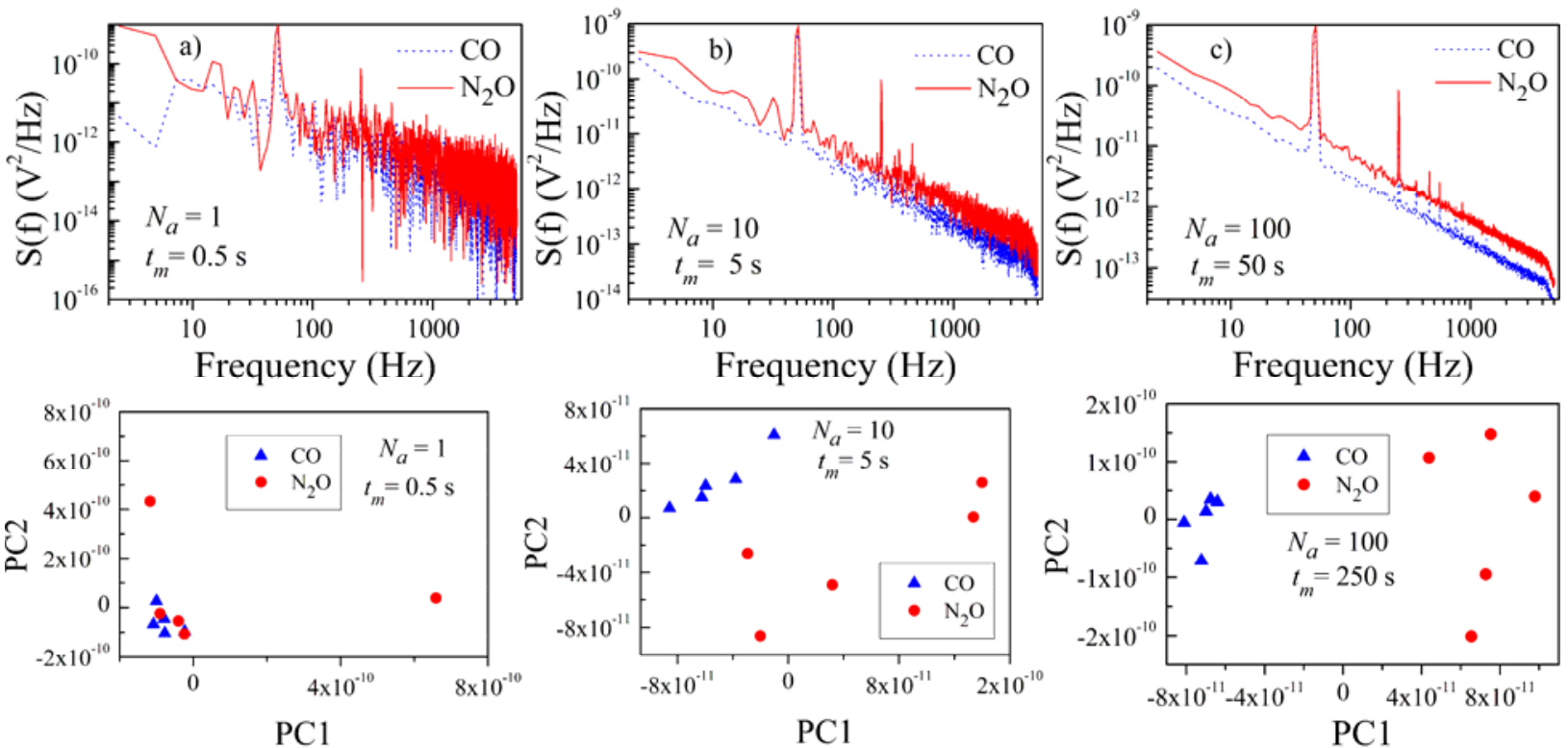

Figure 2 Power Spectral Densities (PSD, top row) and the corresponding PCA maps (bottom row) obtained when varying the data collection duration and averaging factors. Data measured using $20 \mathrm{ppm}$ analyte in synthetic air.

PSDs as input for the PCA analysis. However, clear discrimination was possible when the $N_{a}$ value of 10 was used.

3.2 Effect of gate voltage manipulation Our sensor chip allows for the tuning of a simple multiplexing parameter, that is the gate $(G)$ voltage that can be applied between the source (S) and drain (D) electrodes. In the FES measurement scheme a constant current value is injected through S and D and then the PSD of the resistance (that can be measured between $\mathrm{S}$ and $\mathrm{D}$ ) is derived. The results of tests on the unfunctionalized MWCNT sensor are summarized in Figure 3. for an exposure of $\mathrm{NH}_{3}, \mathrm{~N}_{2} \mathrm{O}$ and $\mathrm{H}_{2} \mathrm{~S}$. The PCA analysis on this sensor, for various gate voltages, showed that practically no discrimination between $\mathrm{NH}_{3}$ and $\mathrm{H}_{2} \mathrm{~S}$ was possible, but importantly, $\mathrm{N}_{2} \mathrm{O}$ could be well differentiated from the previous gases, see Figure 3. A slight effect could be observed here for the gate voltage, $\mathrm{N}_{2} \mathrm{O}$ was best separated from the group to $\mathrm{NH}_{3}$ and $\mathrm{H}_{2} \mathrm{~S}$ at $\mathrm{G}=+50 \mathrm{~V}$. However, the overall effect of gate voltage on the FES selectivity appears to be rather minor.

3.3 Effect of spectral range manipulation $A$ third multiplexing FES parameter can be the size of the spectral window of the PSD that serves as input for the pattern recognition method for the chemical classification of the target gases. The proper choice, in principle, can increase the success rate of the chemical classification and, if the optimal spectral range lies in the higher frequency region, can significantly decrease the measurement time, thus speeding up the classification process. A PCA analysis of the PSDs that were measured after a 20 ppm exposure of the test gases $\left(\mathrm{N}_{2} \mathrm{O}, \mathrm{NH}_{3}\right.$ and $\left.\mathrm{H}_{2} \mathrm{~S}\right)$ can be seen in Figure 4 . The different panels exhibit results when different spectral windows of the acquired PSDs were used as input for the PCA analysis.
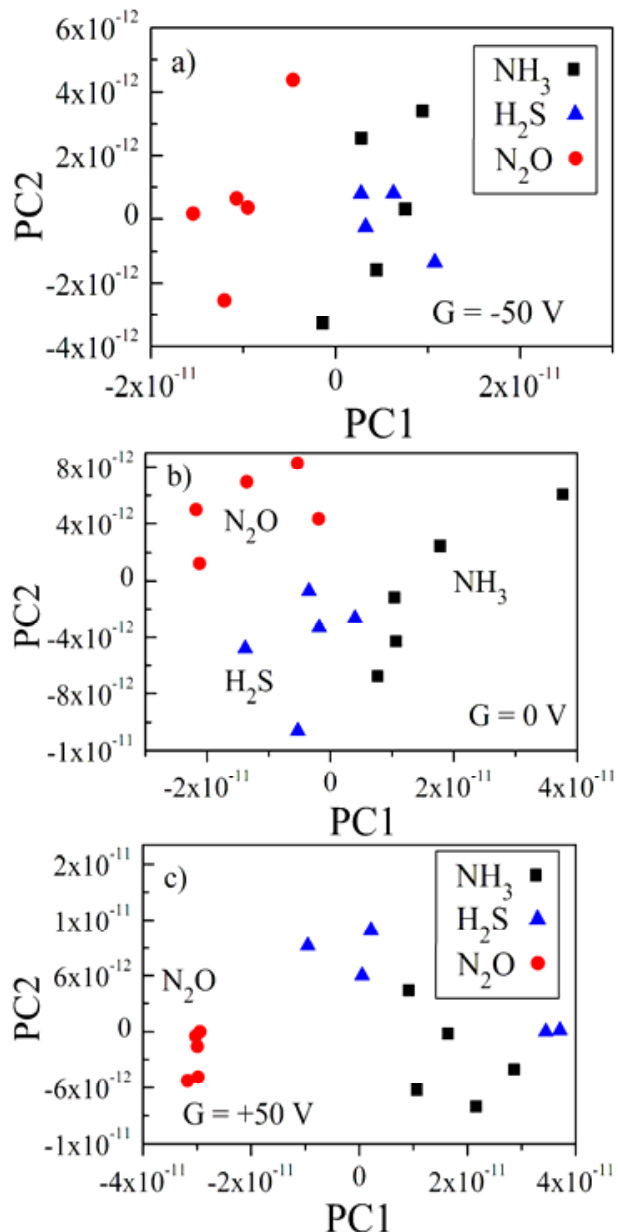

Figure 3. Gate voltage dependence of the chemical selectivity of an MWCNT FES sensor device. 

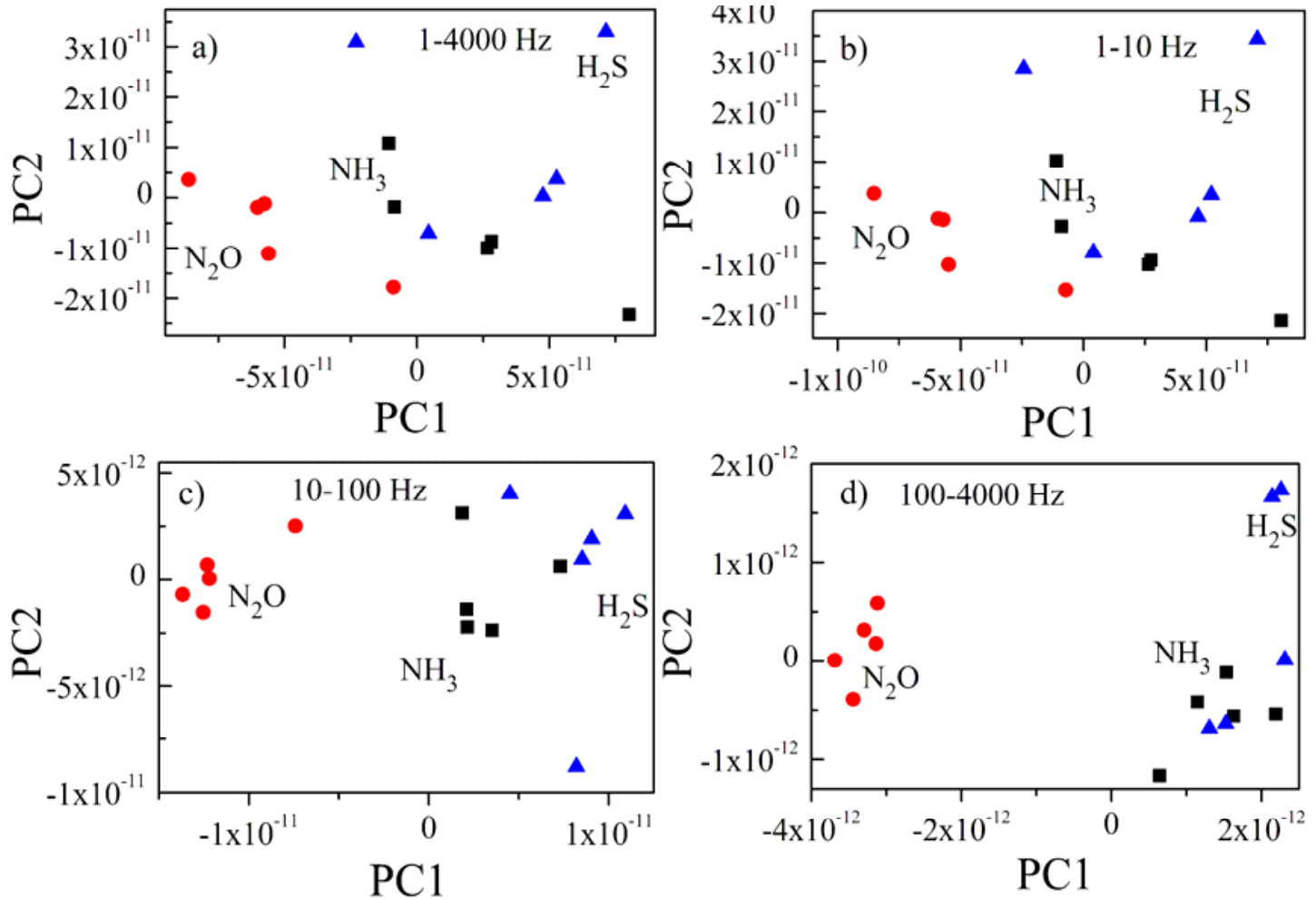

Figure 4 Effect of tuning the PSD frequency window on the chemical selectivity of an MWCNT-FES gas sensor.

This observation is important, since the use of the 10 $100 \mathrm{~Hz}$ spectral range decreases the measurement time by a factor of 10 compared to the use of the whole $(1-4000 \mathrm{~Hz})$ spectral window.

3.4 FES in gas mixture analysis FES measurements with $\mathrm{N}_{2} \mathrm{O} / \mathrm{H}_{2} \mathrm{~S}$ gas mixtures were also performed on both an unfunctionalized MWCNT sensor and a reference amine functionalized SWCNT sensor with total concentrations of $20 \mathrm{ppm}$ of the additives, see Figure 5. It is well known that the extent of CNT surface functionalization can be finely controlled, therefore, it would be possible to create a whole series of CNT FES sensors with e.g. gradually increasing surface hydrophobicity and use this array if a simple MWCNT-SWCNT pair should lack adequate sensing properties [15]. The amine functionalized SWCNT sensor performed better. The $20+0,15+5$ and $0+20 \mathrm{mix}-$ tures (the first and second number mark $\mathrm{N}_{2} \mathrm{O}$ and $\mathrm{H}_{2} \mathrm{~S}$ concentrations in ppm as noting the mixtures) can be clearly classified. The $10+10$ and $5+15$ mixtures have however some mutual overlapping, these two latter mixtures clearly cluster differently than the other three mixtures. Moreover, there is a tendency of the PC1 component to increase as increasing the $\mathrm{H}_{2} \mathrm{~S}$ concentration in the mixtures. Such tendency can not be seen for the unfunctionalized MWCNT sensor however, the mixtures cluster differently, though not such a pronounced way as for the SWCNT sensor. Nevertheless, the measurements show that FES with CNT sensor is capable of differentiating between gas mixtures.
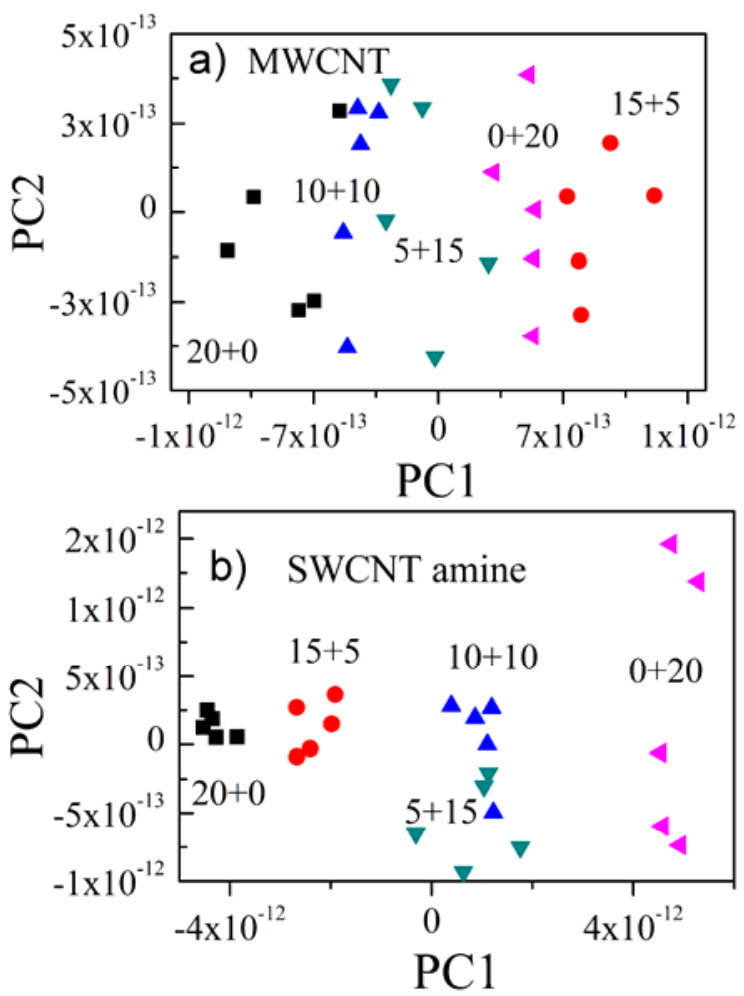

Figure 5. Performance of CNT FES sensors in gas mixture analysis 
4. Conclusion In this contribution we reviewed three different possibilities for improving the chemical selectivity of an unfunctionalized MWCNT film used for gas sensing in a Fluctuation Enhanced Sensing setup. The effect of gate voltage changes on the selectivity is marginal. On the other hand, chemical differentiation can be improved radically by increasing the data collection duration and using the right spectral window (100 averages and 10-100 Hz, respectively). Preliminary results on combining chemical selectivity with quantitative analysis possibilities indicates that the FES method is capable of extracting qualitative and quantitative information from the sensor noise simultaneously. Whereas the type of carbon nanotubes used is not very important as long as only qualitative information is sought, it becomes a major factor in quantitative gas mixture analysis. Our first results indicate that aminefunctionalized single-wall carbon nanotubes perform better in this task than unfunctionalized multi-wall carbon nanotubes.

Further studies, carried out at both experimental and theoretical levels, are in progress to gain insight into the mechanisms determining the analyte-specific noise patterns in FES experiments $[16,17]$.

Acknowledgements The financial support of the European Commission (FP6 STREP \#17310 „SANES”) and the Hungarian Scientific Research Fund (OTKA) through project NNF78920 is acknowledged. Computational resources for theoretical support are provided by the SGI/IZO-SGIker UPV/EHU (Arina cluster).

\section{References}

[1] E. Comini, Anal. Chim. Acta 568, 28, (2006).

[2] Q. Wan, Q.H. Li, Y.J. Chen, T.H. Wang, X.L. He, J.P. Li, and C.L. Lin, Appl. Phys. Lett. 84, 3654 (2004).

[3] P.A. Lieberzeit, and F.L. Dickert, Anal. Bioanal. Chemi. 387, 237 (2007)..

[4] T. Simon, N. Barsan, M. Bauer, and U. Weimar, Sens. Act. B - Chemical, 73, 1 (2001).

[5] N. Sinha, J.Z. Ma, and J.T.W. Yeow, J. Nanosci. Nanotech. 6, 573 (2006).

[6] T. Zhang, S. Mubeen, E. Bekyarova, B. Y. Yoo, R. C. Haddon, N. V. Myung, and M. A. Deshusses, Nanotechnology 18 (2007).

[7] J. Zhao, A. Buldum, J. Ha, and J. P. Lu, Nanotechnology 13, 195 (2002).

[8] C. Cantalini, L. Valentini, I. Armentano, J.M. Kenny, L. Lozzi, and S. Santucci, J. Eur. Ceram. Soc., 24, 1405 (2004).

[9] H.E. Katz, Electroanalysis, 16, 1837 (2004).

[10] S. Kim, S. Pal, P.M. Ajayan, T. Borca-Tasciuc, and N. Koratkar, J. Nanosci. Nanotech. 8, 416 (2008).

[11] P. Heszler, Z. Gingl, R. Mingesz, A. Csengeri, H. Haspel, A. Kukovecz, Z. Konya, I. Kiricsi, R. Ionescu, J. Maklin, T.
Mustonen, G. Toth, N. Halonen, K. Kordas, J. Vahakangas, and H. Moilanen, phys.stat.sol.b 245, 2343 (2008).

[12] L. B. Kish, R. Vajtai, and C. G. Granqvist, Sens. Actuators B 71, 55 (2000).

[13] H. Haspel, R. Ionescu, P. Heszler, A. Kukovecz, Z. Konya, Z. Gingl, J. Maklin, T. Mustonen, N. Halonen, K. Kordas, R. Vajtai, and P.M. Ajayan, phys.stat.sol.b 245, 2339 (2008).

[14] J. Maklin, T. Mustonen, N. Halonen, G. Toth, K. Kordas, J. Vahakangas, H. Moilanen, A. Kukovecz, Z. Konya, H. Haspel, Z. Gingl, P. Heszler, R. Vajtai, and P.M. Ajayan, phys.stat.sol.b 245, 2335 (2008).

[15] T. Kanyo, Z. Konya, A. Kukovecz, F. Berger, I. Dekány, and I. Kiricsi, Langmuir 20, 1656 (2004).

[16] J. Maklin, N. Halonen, Y. Pouillon, J.M. García Lastra, K. Kordas, A. Rubio, to be published.

[17] P. Heszler, Y. Pouillon, A. Rubio, to be published. 